\title{
Synthesis of Polyesters Containing the [60]Fullerene Moiety in the Main Chain
}

\author{
Masumi Taki, Shoji Takigami, Yuriko Watanabe, Yosuke NaKamura, \\ and Jun NishimURA ${ }^{\dagger}$ \\ Department of Chemistry, Gunma University, \\ 1-5-1 Tenjin-cho, Kiryu 376, Japan
}

(Received June 2, 1997)

\begin{abstract}
Fullerene bisphenol 1 was allowed to react with equimolar amounts of dibasic acid dichlorides at room temperature, to afford linear polyesters 3 and $\mathbf{4}$ containing the [60]fullerene moiety in the main chain. These polyesters were characterized by micro ATR-IR spectroscopy and TG analysis. They were soluble in $N, N$-dimethylformamide (DMF) and weight-average molecular weight $\left(M_{w}\right)$ was determined by GPC.

KEY WORDS [60]Fullerene / Polyester / Copolymer / Fourier Transform Infrared Spectroscopy /

Thermogravimetry / Gel Permeation Chromatography /
\end{abstract}

[60]Fullerene has unique properties, such as marvelous electron affinity, originated from its special nongraphite $\pi$-systems. Many researchers have focused on the materials science of [60]fullerene. ${ }^{1,2}$ Fullerene polymerization has been investigated for this reason, but many difficulties still remain to be solved. For instance, a $[2+2]$ cyclization between adjacent [60]fullerenes, having many reactive sites, affords highly cross-linked, insoluble homopolymer which cannot be molded or even handled conveniently. ${ }^{3-7}$ To avoid such difficulties and prepare soluble fullerene polymers, copolymerization is used.

There are two general fullerene-containing copolymers $^{2}$ : 1) Bracelet polymers to which fullerenes are attached, but are not part of the backbone and 2) pearlnecklace polymers whose fullerenes are part of the backbone.

Comparing these copolymers, pearl-necklace copolymers may retain fullerene properties in the bulk state, because fullerene moieties line up densely and alternatively on the backbone. However, most pearl-necklace fullerene copolymers could not be dissolved in any solvent at all because of their cross-linking, and molecular weight was difficult to determine. ${ }^{8}$ Moreover, the conditions of polymerization in most cases require high temperature and/or strong reagents. ${ }^{9,10}$ Thus, few studies have been reported on the synthesis and characterization of copolymers.

Since we recently developed a reliable and selective synthetic method for [60] fullerene derivative 1 possessing two phenol moieties on the nanoparticle surface, ${ }^{11}$ we were prompted to take the advantage of high reactivity on the hydroxy groups, as a monomer, and make soluble linear pearl-necklace type polyesters (Scheme 1). This paper reports the polymerization of the soluble alternative pearl-necklace polyesters under mild conditions and characterizes their properties.

\section{EXPERIMENTAL}

\section{Materials}

The most readily available monomer, $(\mathrm{A}, \mathrm{H})$-regioisomer $1,{ }^{12}$ was isolated and purified by the reported method. ${ }^{11}$ Isophthaloyl chloride, terephthaloyl chloride, and sebacoyl chloride were of highest grade, and other chemicals were of reagent grade. They were obtained commercially and used without further purification.

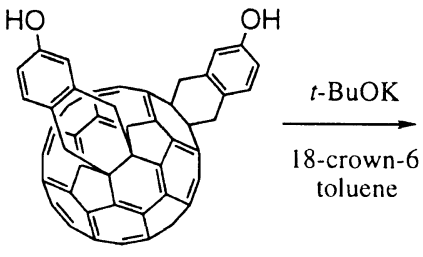

(A, H)-bisadduct

1

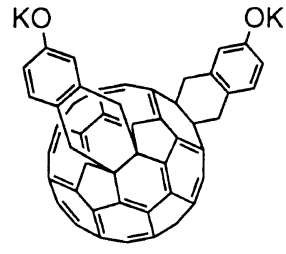

soluble in water 2

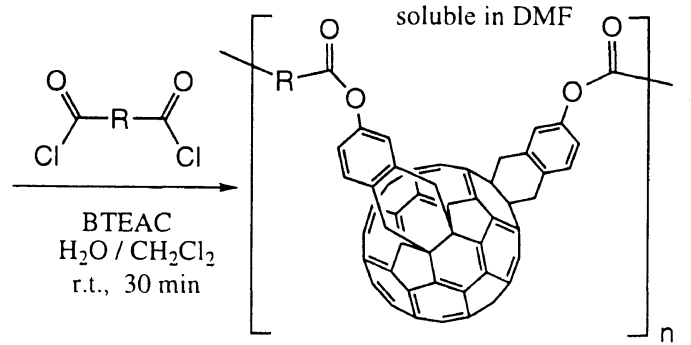

(1)

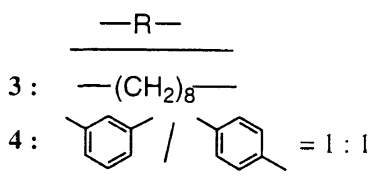

Scheme 1.

† To whom correspondence should be addressed. 
Synthesis of [60]Fullerene Dianion Salt 2

A mixture of potassium tert-butoxide $(22 \mathrm{mg})$ and 18-crown-6 $(53 \mathrm{mg})$ in toluene $(10 \mathrm{~mL})$ was added to monomer $1\left(10 \mathrm{mg}, 1.0 \times 10^{-5} \mathrm{~mol}\right.$, dissolved in $10 \mathrm{~mL}$ of toluene) at room temperature. Soon, a dark brown salt precipitated. The precipitate was collected by filtration, washed with toluene $(50 \mathrm{~mL} \times 2)$, and dried completely under reduced pressure to remove toluene. The obtained dark brown fullerene salt $2(10 \mathrm{mg})$ was subjected to the following reactions without further purification.

\section{Polycondensation Reaction}

To a solution $(10 \mathrm{~mL})$ of dibasic acid dichloride $(20 \mathrm{mg})$ containing benzyl triethylammonium chloride (trace) as a phase transfer catalyst was added [60]fullerene salt $\mathbf{2}$ $(10 \mathrm{mg})$ dissolved in water $(5 \mathrm{~mL})$ at room temperature. The reaction mixture was stirred vigorously at room temperature for $30 \mathrm{~min}$. The crude reaction mixture was poured into ethanol $(50 \mathrm{~mL})$ and the product was collected by filtration. It was washed with water, ethanol, and then tetrahydrofuran (THF), and dried in vacuo at room temperature, affording fullerene-containing homopolymer $3(7 \mathrm{mg}, 60 \%$ yield ) or copolymer $4(6 \mathrm{mg}, 60 \%$ yield). Without 2 , no polymeric material was obtained from the reaction mixture. Sebacoyl chloride was used as a pure monomer, but isophthaloyl chloride and terephthaloyl chloride were mixed at $1: 1$ and used as a monomer mixture, because pure phthaloyl chlorides often give insoluble, highly crystalline homopolymers.

\section{Fourier Transform Infrared (FT-IR) Spectroscopy}

IR spectra were measured using a Nicolet Magna 750 FT-IR spectrophotometer equipped with an infrared microscope. The fullerene polyesters were analyzed by ATR method. A ZnSe polarizer was used in the case of high refractive index material.

\section{Molecular Weight Measurement}

Weight-average molecular weights $\left(M_{w}\right)$ of polymers were determined by high performance liquid chromatography (Tosoh HLC-8120GPC) using a column (Tosoh TSKgel $\alpha-M, 7.8 \mathrm{~mm}$ i.d. $\times 30 \mathrm{~cm} \times 2$ ). The mobile phase was $10 \mathrm{mM}-\mathrm{LiBr}$ in dimethylformamide. Measurement was carried out using a UV detector $(333 \mathrm{~nm})$ at $40^{\circ} \mathrm{C}$ and flow rate of $1.0 \mathrm{~mL} \mathrm{~min}^{-1}$. The column was calibrated using polystyrene standard samples of defined molecular weights.

\section{Thermogravimetry}

Thermal decomposition behavior of homopolymer 3 and copolymer 4 was analyzed by a Perkin-Elmer TGA-7 balance. The samples ( $c a .1 \mathrm{mg}$ ) were heated from room temperature to $800^{\circ} \mathrm{C}$ at $10^{\circ} \mathrm{C} \mathrm{min}^{-1}$ under a nitrogen atmosphere.

\section{RESULTS AND DISCUSSION}

[60]Fullerene bisphenol $\mathbf{1}$ itself could hardly be dissolved in water because of its strong hydrophobicity. When 1 was converted into salt $\mathbf{2}$ by the treatment with potassium tert-butoxide in the presence of 18 -crown- $6,{ }^{13}$ solubility in water dramatically increased. The strong

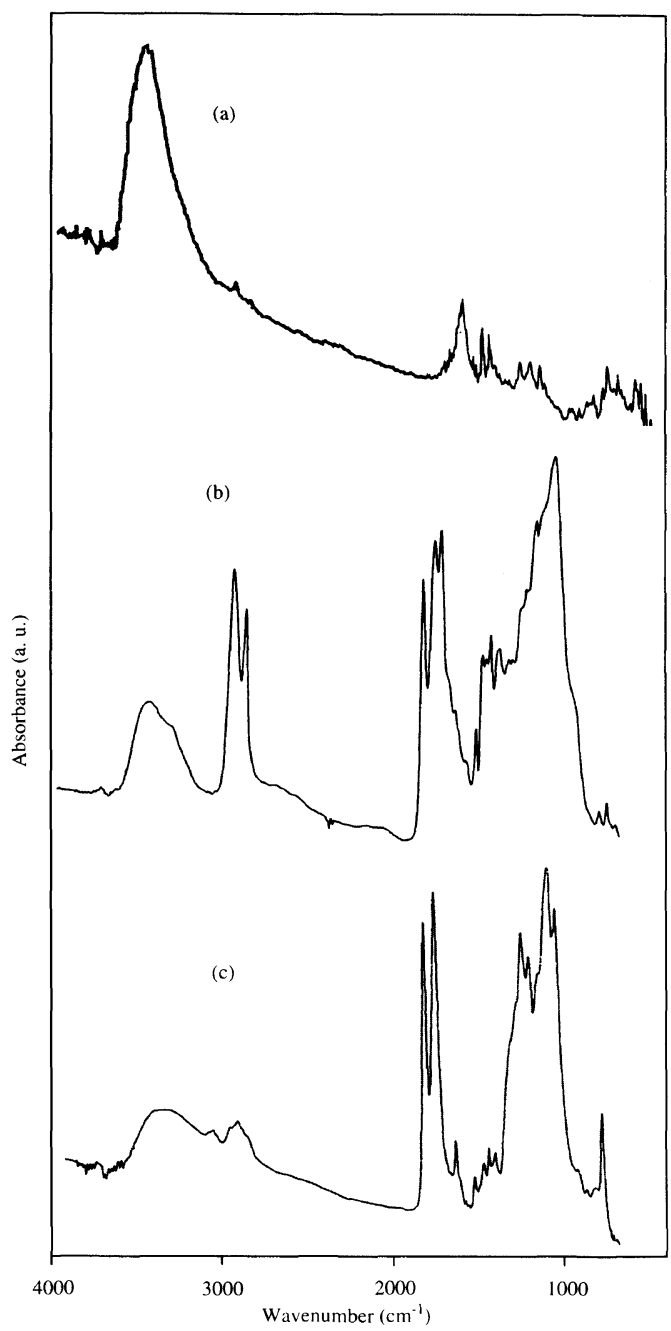

Figure 1. FT-IR spectra of monomer (a) 1, (b) homopolymer 3, and (c) copolymer 4 .

hydrophilicity of salt $\mathbf{2}$ allows us to obtain desirable polyesters 3 and 4 by surface condensation polymerization under mild conditions (Scheme 1).

The reaction mixture was separated into water and organic phases at the early stage of polycondensation. The interface between phases suspended gradually within $10 \mathrm{~min}$. Finally, decoloration of the water phase occurred with consumption of the fullerene monomer, giving aggregated thread-like brown product 3 or 4 .

Figure 1 shows FT-IR spectra of products 3 and 4. The $\mathrm{OH}$-stretching broad band, characteristic of bisphenol-type monomer 1, disappeared and new $\mathrm{C}-\mathrm{O}-\mathrm{C}$ typical bands were observed in the finger region from 1000 to $1500 \mathrm{~cm}^{-1}$. The intense bands which appeared around $1700-1800 \mathrm{~cm}^{-1}$ are characteristic of the carbonyl stretching vibration. Condensation occurred and polyesters formed under such mild conditions.

Figure 2 shows the GPC chromatogram of product 3 with the elution times. A strong peak was observed at 18 min with several small peaks appearing at longer retention times. The small peaks of less-than-1000 molecular weights were considered to be due to byproducts, and thus neglected. Figure 3 shows the GPC chromatogram with the molecular weights from 14.5 to $19.3 \mathrm{~min}$. The starting point of peak was 200000 and the peak top was 4000 . The weight-average molecular weight 


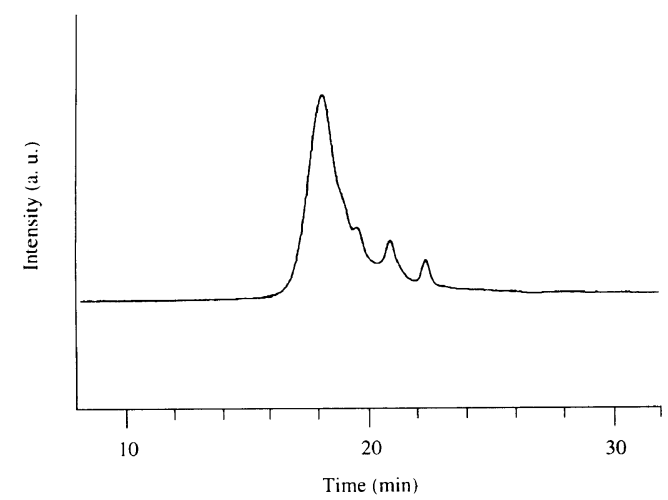

Figure 2. GPC chromatogram of homopolymer 3 with the elution times as the horizontal axis.

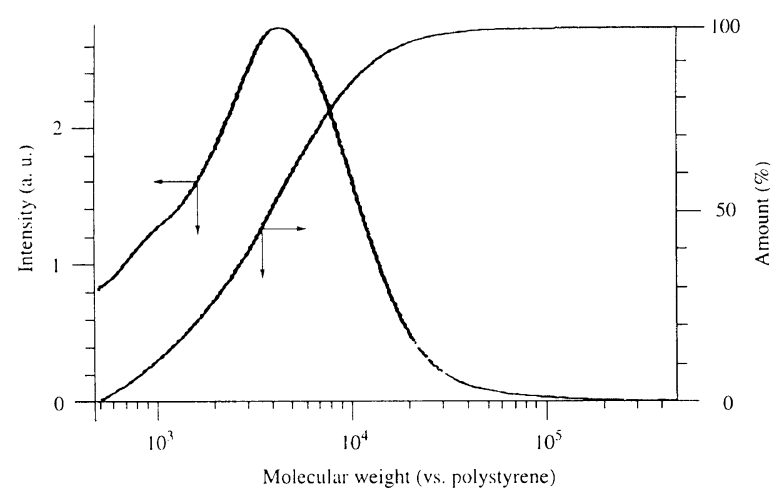

Figure 3. GPC chromatogram of homopolymer $\mathbf{3}$ with the molecular weights as the horizontal axis.

$\left(M_{w}\right)$ of product 3 was determined to be 6000 and the polydispersity $\left(M_{w} / M_{n}\right)$ was 2.6 . Although most of the products were oligomers, such polyesters with high molecular weights were also synthesized even at room temperature.

Figure 4 shows the TG curves for 3 and 4 . A double-stage decomposition reaction was observed in the case of homopolymer 3. The mass loss at the first stage was $65 \%$, which was observed from 160 to $420^{\circ} \mathrm{C}$. This is attributable to the ring opening reaction of the cyclohexene moiety or retro-Diels-Alder reaction, followed by the sublimation or vaporization of fragments. The second stage was observed from 460 to $540^{\circ} \mathrm{C}$ and no residue was found over $540^{\circ} \mathrm{C}$. This means that polyester $\mathbf{3}$ fully decomposed and fragments were wholly sublimated or vaporized. An overlapping multistage decomposition was observed in the case of copolymer 4. A more complicated decomposition thus occurred for this copolymer. Aromatic polyesters have higher thermostability than aliphatic, and this general behavior is coincidence with the present experimental results. Therefore, these obvious differences between polyesters 3 and $\mathbf{4}$ are due to the structures of the polymers originated from the monomer units.

We established a methodology for preparing linear and soluble polyesters containing [60]fullerene moieties alternatively in the main chain. Polymerization efficiently occurred under very mild conditions, thus avoiding any complicated side reactions causing troublesome cross-

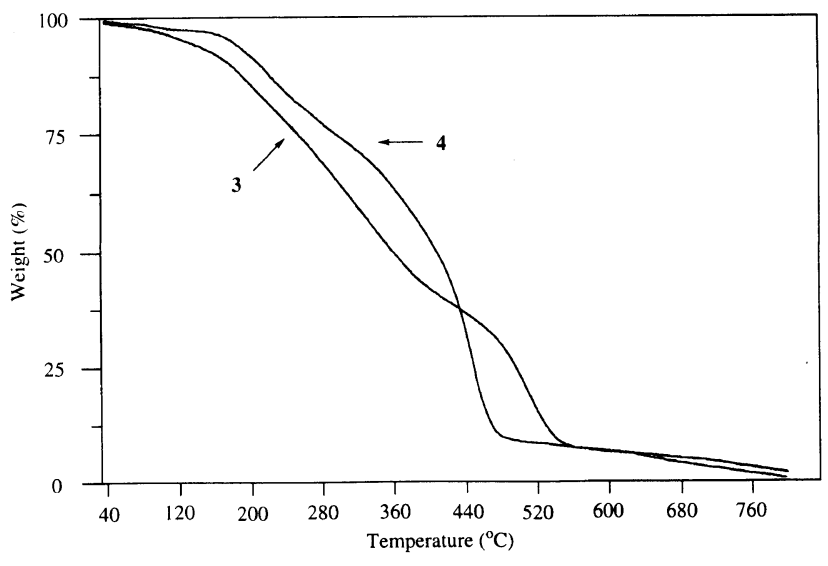

Figure 4. TG curves for homopolymer 3 and copolymer 4 .

linking etc. According to GPC experiments, some products were found to have quite high molecular weights, although most were oligomers. Micro ATR-IR measurements and TG analysis were useful for the characterization of fullerene polymers. We are now investigating other properties of fullerene polyesters.

Acknowledgments. This work was partly supported by Grant-in-Aid for Scientific Research on Priority Area, "New Polymers and Their Nano-Organized Systems" (No. 277 / 08232208), from the Ministry of Education, Science, Sports, and Culture of Japan and the Nishida Research Fund for Fundamental Organic Chemistry. One of the authors (M.T.) was supported by Research Fellowships (DC1) of the Japan Society for the Promotion of Science for Young Scientists. GPC analysis was performed by Tosoh Co., Ltd. The authors are grateful to Mr. Kiyoshi Miyashita for obtaining ATRIR spectra, and to Mr. Takashi Kondo for TG analysis at Gunma Prefectrual Ind. Tech. Res. Laboratory.

\section{REFERENCES}

1. Y. S. Obeng and A. J. Bard, J. Am. Chem. Soc., 113, 6279 (1991).

2. C. A. Mirkin and W. B. Caldwell, Tetrahedron, 52, 5113 (1996).

3. J. E. Fischer, Science, 264, 1548 (1994).

4. S. Pekker, A. Janossy, L. Mihaly, O. Chauvet, M. Carrard, and L. Forro, Science, 265, 1077 (1994).

5. A. M. Rao, P. Zhou, K. Wang, G. T. Hager, J. M. Holden, Y. Wang, W. Lee, X. Bi, P. C. Eklund, D. S. Cornett, M. A. Duncan, and I. J. Amster, Science, 259, 955 (1993).

6. Y. Wang, J. M. Holden, X. Bi, and P. C. Eklund, Chem. Phys. Lett., 217, 413 (1994)

7. P. W. Stephens, G. Bortel, G. Faigel, M. Tegze, A. Janossy, S. Pekker, G. Oszlanyi, and L. Forro, Nature, 370, 636 (1994).

8. D. A. Loy and R. A. Assink, J. Am. Chem. Soc., 114, 3977 (1992).

9. S. Shi, K.C. Khemani, Q. Li, and F. Wudl, J. Am. Chem. Soc., 114, 10656 (1992)

10. A. Gügel, P. Belik, M. Walter, A. Kraus, E. Harth, M. Wagner, J. Spickermann, and K. Müllen, Tetrahedron, 52, 5007 (1996).

11. M. Taki, S. Sugita, Y. Nakamura, E. Kasashima, E. Yashima, Y. Okamoto, and J. Nishimura, J. Am. Chem. Soc., 119, 926 (1997).

12. For the nomenclature, see Y. Nakamura, M. Taki, and J. Nishimura, Chem. Lett., 703 (1995).

13. W. Bidell, R. E. Douthwaite, M. L. H. Green, A. H. H. Stephans, and J. F. C. Turner, J. Chem. Soc., Chem. Commun., 1641 (1994). 